\title{
Lippenbekenntnisse - wie Versprechen (nicht) eingehalten werden
}

\begin{abstract}
Stärkung und Förderung der Grundversorgung und der peripheren Medizin, bessere Abgeltung der intellektuellen Leistung, Erhaltung des Praxislabors, gleiche Honorierung für gleiche Leistung - das sind alles Schlagworte, die im Gesundheitswesen gebraucht werden. Schöne Worte wenn jedoch Taten ausbleiben, sind sie nur reine Lippenbekenntnisse. Obwohl in der ambulanten Praxismedizin die Kosten Jahr für Jahr unter dem schweizerischen Durchschnitt ansteigen, sind die Massnahmen zur Kosteneindämmung vorwiegend gegen die praktischen Ärzte gerichtet: Die Revision der Analysenliste, die Reduktion des Vertriebsanteils in der Medikamentenabgabe, die Taxpunktwertanpassung im UV/IV/MV-Bereich, die Umsetzung der LeiKoV (Leistungskostenvereinbarung) im KVGBereich sind einige Beispiele dafür.
\end{abstract}

\section{Praxislabor}

Die Revision der Analysenliste ist notwendig und als solche auch unbestritten. Die Beurteilung müsste nach WZW (Wirksamkeit, Zweckmässigkeit und Wirtschaftlichkeit) erfolgen. In der aktuellen Revision werden vom Bundesamt für Gesundheit (BAG) aber nur die wirtschaftlichen Positionen überprüft. Es spricht bei den Grundlagenpapieren zur Revision von einem hohen Gewinn und einer Querfinanzierung der Praxis durch die Gewinne aus dem Praxislabor! Unsere Berechnungen auf der Basis der Workflowstudie zum Praxislabor mit einem Vollkostenmodell ergeben bei der vorgesehenen Reform ganz andere Zahlen: nämlich eine Absenkung von 36\%, was für kein Praxislabor mehr kostendeckende Preise ergibt!

Die Version Beta, die das BAG im Juni in die Vernehmlassung gab, hat gezeigt, dass das Praxislabor in keiner Art und Weise korrekt abgebildet wird. Wir haben in unserer Anhörungsantwort mit Fakten, betriebswirtschaftlichen Berechnungen und konstruktiven Vorschlägen auf diese Probleme aufmerksam gemacht und eine separate Neuberechnung des Praxislabors unter Beizug der Experten der FMH gefordert. Seither ist gar nichts passiert! In der Version Gamma, die in der Analysenkommission des BAG am 22. September 2008 besprochen wurde, finden sich nur geringfügige Änderungen gegenüber der Version Beta. Unsere Anliegen und Forderungen wurden in keiner Art und Weise berücksichtig. Im Gegenteil: Peter Indra, Vize- direktor des BAG, bezichtigt uns in einem Interview in der Berner Zeitung vom 22. September 2008 der Ignoranz und meint, wir hätten falsch gerechnet und die Präsenztaxe nicht berücksichtigt. Dies haben wir aber sehr wohl gemacht. Herr Indra könnte das in unserer Anhörungsantwort nachlesen, die seit Juli auf seinem Pult liegt. Die Präsenztaxe, die mit einem Frankenbetrag pro Laborsitzung die Nachteile der Praxislabors ausgleichen soll, ist aber kein valabler Ersatz. Den anderen Produktionsbedingungen im Praxislabor in Bezug auf Geräte, Reagenzien und Serienlängen kann nur mit einem separaten Tarif entsprochen werden.

Die vorliegende Version Gamma wird zur Folge haben, dass eine grosse Anzahl der Praxislaboratorien geschlossen werden muss. Der Bund spart auf der Ebene des Praxislabors keinen Rappen, die Kosten werden nur verlagert und generieren Mehrkosten. Und ein gut und effizient funktionierendes System wird zerstört. Leidtragende werden die Patienten sein. Einmal mehr wird über Tarife und Preise Strukturpolitik betrieben, ohne dass man wirklich dazu steht. Unter diesen Umständen ist das Bekenntnis des BAG zur Erhaltung des Praxislabors und zur Förderung sowie Stärkung der Grundversorgung ein reines Lippenbekenntnis.

\section{Massnahmen}

Wir sind auf breiter Ebene am Ball und bringen die Anliegen der Ärzteschaft in Verhandlungen und Diskussionen ein. Wir kämpfen auf technischer Ebene und im politischen Bereich für den Erhalt des Praxislabors. Ebenso bereiten wir auf verschiedenen Ebenen Massnahmen vor, um unseren Forderungen Achtung zu verschaffen. Die auf breiter Front geplanten Massnahmen des BAG zur Schwächung der Grundversorgung und der peripheren Medizin werden wir keinesfalls widerstandslos akzeptieren! Unterstützen Sie auch die Briefaktion von SGAM, SGIM, SGP, anderer betroffenen Fachgesellschaften und der FMH. Weitere Informationen finden Sie in dieser Ausgabe der SÄZ auf Seite 1758.

Dr. med. Ernst Gähler, Vizepräsident der FMH, Verantwortlicher Ressort Tarife und Verträge 